\title{
Combinatorial Auction with Time-Frequency Flexibility in Cognitive Radio Networks
}

\author{
Mo Dong, Gaofei Sun, Xinbing Wang \\ Department of Electronic Engineering \\ Shanghai Jiao Tong University, China \\ Email:\{dongmo007, sgf_hb, xwang8\}@sjtu.edu.cn
}

\author{
Qian Zhang \\ Dept. of Comp. Sci. and Engin \\ HK Univ. of Sci. and Tech., HongKong \\ Email:qianzh@cs.ust.hk
}

\begin{abstract}
In this paper, we tackle the spectrum allocation problem in cognitive radio (CR) networks with time-frequency flexibility consideration using combinatorial auction. Different from all the previous works using auction mechanisms, we model the spectrum opportunity in a time-frequency division manner. This model caters to much more flexible requirements from secondary users (SUs) and has very clear application meaning. The additional flexibility also brings theoretical and computational difficulties. We model the spectrum allocation as a combinatorial auction and show that under the time-frequency flexible model, reaching the social welfare maximal is NP hard and the upper bound of worst-case approximation ratio is $\sqrt{m}$, $m$ is the number of time-frequency slots. Therefore, we design an auction mechanism with near-optimal winner determination algorithm, whose worst-case approximation ratio reaches the upper bound $\sqrt{m}$. Further we devise a truthful payment scheme under the approximation winner determination algorithm to guarantee that all the bids submitted by SUs reflect their true valuation of the spectrum. To further address the issue and reach optimality, we simplify the general model to that only frequency flexibility is allowed, which is still useful, and propose a truthful, optimal and computationally efficient auction mechanism under modified model. Extensive simulation results show that all the proposed algorithms generate high social welfare as well as high spectrum utilization ratio. What's more, the actual approximation ratio of near-optimal algorithm is much higher than the worst-case approximation ratio.
\end{abstract}

\section{INTRODUCTION}

With the ongoing growth of wireless communication services, the demand for radio spectrum increases dramatically. However, the spectrum resource is scarce because of the longexisted rigid licence policy. Former studies [1] [3] show that from a time-spatial view, there are a large number of underutilized spectrum holes in the current wireless networks. To overcome the low-efficiency, cognitive radio (CR) networks was proposed [4]. In the CR networks, the original licensed operators are called primary users (PUs) and the users who want to exploit the spectrum opportunity are called secondary users (SUs). To implement CR, an efficient, robust and fast dynamic spectrum access (DSA) mechanism is needed to bring the spectrum opportunity from PUs to SUs and provide enough incentive for PUs. Extensive works have been carried out on this topic and most of them cooperate economical tools such as game theory [6], contract theory [7], auction [8], commodity pricing [5] [19] and etc. Among those mechanisms, auction is preeminent because of its fairness, efficiency and valuation independence [9]. Various auction mechanisms have been proposed to cope with the DSA problem [11] [13] [15].

In general, the DSA auction mechanisms can be divided into two categories: periodical and online auction. Periodical auction means that the spectrum opportunity is sold periodically by the auctioneer (a single PU or an agent called Primary Operator(PO) of many PUs) and the interval between different auctions is relatively long (30 minutes or longer). As for online auction, the auction process is done on every new arrival of SU. For both online and periodical auction, it is critical to guarantee an economical property called truthfulness [9], that is to say the bids submitted by the SUs reflect their true valuation for the spectrum opportunity. The property is crucial in that if any SU can lie to gain a larger utility through auction, efficiency and fairness of auction mechanism can not be achieved. In [17], Gandhi proposed a general centralized periodical auction mechanism for spectrum allocation in cognitive radio networks. Contrast to the centralized method, Chen [18] designed a distributed algorithm under an extended system model. To extend the auction to deal with muti-seller problem, Gao [16] proposed a truthful DSA mechanism based on progressive auction. In [14], Gopinathan not only considered single round auction but also extended it to repeated auction with consideration of fairness and truthfulness as well. All the above auction mechanisms are periodical auction and online auction is also widely used [15] [20]. In [21], not only preemption but also a time-cheating problem was considered in online fashion. Despite all the previous works, a significant problem is not fully addressed: the time-frequency flexibility of SUs' requirements.

To illustrate the time-frequency flexibility of SUs' requirements clearly, we will firstly give a sketch of our system model. In our model, a primary operator $(P O)$ presents as an auctioneer. Periodically, this PO contacts with the spectrum opportunity data center to obtain the free spectrum from PUs. The PO then divides the spectrum opportunity by frequency and time as shown in Figure 1. We can see that colored piece in Figure 1 means a spectrum opportunity from $t_{0}$ to $t_{0}+\Delta t$ at frequency $f_{0}$ to $f_{0}+\Delta f$. We denote this piece of spectrum opportunity as a slot. The reason for the division lies in the heterogeneity and time-frequency flexibility of SUs' requirements as shown in Figure 1. In Figure 1, SU1's requirement is varying over time and frequency. At the 


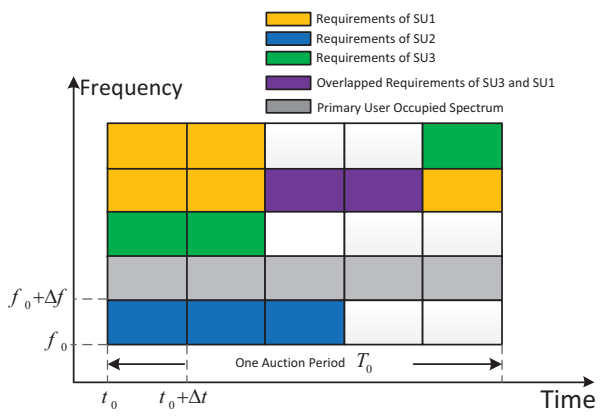

Fig. 1. SUs' Heterogenous Requirements

beginning of the period, he may download some files from the Internet, but after the anticipated downloading time, the extra bandwidth is not necessary because he only wants to browse some web pages. As for SU2, he only wants to check his mail and therefore, the period for him is too long that he only needs a part of the period over constant frequency. SU3 is a secure device so that he needs to jump from one frequency to another in the same auction period. SU3's requirement is partially overlapped with SU1's. As far as our knowledge, all the previous works on periodical auction considered different SUs' requirements as fixed over the auction period and the SUs can only buy the entire time span of fixed frequency or nothing. The time-frequency two dimensional heterogeneity and flexibility of SUs' requirements are not fully considered.

In our paper, we model every slot of the spectrum opportunity as an individual good to be sold. We allow a buyer to submit a bid consisting of an arbitrary bundle of the goods and the value he is willing to pay for them to the auctioneer. Therefore, the time-frequency flexible requirements can be fulfilled. This kind of auction is called combinatorial auction in economics [22]. To make this auction framework actually work, our paper aims at solving two critical problems:1.Design winner determination mechanism which can run in a tolerable time and with a high efficiency (maximal social welfare); 2. Design a payment policy to guarantee truthfulness. However, we will see in the section III, that the winner determination problem under the fully flexible requirement model is NP hard. Therefore, we analyze the upper bound of polynomial time algorithms' worst-case approximation ratio and find a polynomial-time approximation algorithm with an worst-case approximation ratio reaching the upper bound. Based on the winner determination algorithm, we propose a payment scheme to guarantee the truthfulness in the process of bids submission. To further address the problem, in section IV we put an additional restriction on the SUs' requirements and find an optimal algorithm for winner determination problem under this simplified model. For the simplified model, we provide a truthful payment mechanism based on VCG mechanism design.

To our knowledge, our work has the following key contributions:
- We model the PO's spectrum stack in a new way by considering the problem of heterogeneous requirements with time-frequency flexibility from SUs, and formulate the problem as combinatorial auction problem.

- To solve proposed the combinatorial auction problem, we firstly prove the winner determination problem under the time-frequency flexible auction model is NP hard and then, design a novel auction mechanism consisting of a polynomial time and near-optimal winner determination algorithm and a novel payment mechanism that guarantees the truthfulness of the auction mechanism.

- To further address the problem and reach the optimal solution, we reduce the general time-frequency flexible model to only frequency flexible, give an optimal solution to the winner determination problem under simplified system model and utilize the VCG mechanism to design a payment mechanism that guarantees truthfulness.

The rest of this paper is organized as follows. In Section II, we describe the general framework of cognitive radio networks and propose time-frequency two dimensional flexible system model. In Section III, we provide an auction mechanism that is computationally efficient, near-optimal and truthful under the two dimensional flexibility. In Section IV, we will simplify the general system model without losing application meaning and propose an optimal, computationally efficient and truthful auction mechanism. Section $\mathrm{V}$ provides extensive simulation results show the performance of our proposed algorithms. In section VI, we give the conclusion and show some possible future works.

\section{System Model And Problem Formation}

In this paper, we consider the whole CR network model as Figure 2. The model is a four-layer model:the first layer is the PU networks and they are the source of spectrum opportunity; the second layer contains the spectrum data centers and they collect the information of spectrum opportunity from the PUs via various ways for example, large-scale sensor networks[29]; the third layer lies the primary operators which contact the data center for the spectrum opportunity and sell them to SUs in the fourth layer. In our paper, we only concentrate on the problem that how the PO sells the acquired spectrum to SUs. Therefore, from PO's perspective the spectrum is known and stable during one auction interval. As shown in Figure 1, the spectrum opportunity owned by PO is divided by time and frequency. We denote the entire spectrum opportunity of PO as $S$. We now denote the time interval as $\Delta t$ and the frequency interval as $\Delta f$ and every $\Delta t \times \Delta f$ spectrum that starts from $\left(t_{0}, f_{0}\right)$ as $s_{\left(t_{0}, f_{0}\right)}$. We assume that in one auction period, the spectrum opportunity remains unchanged and there are totally $m$ slots. Therefore, we can map the $\left(t_{0}, f_{0}\right)$ to $i$, where $i \in\{1, \ldots, m\}$. Hence, we change the notation of $s_{\left(t_{0}, f_{0}\right)}$ to $s_{i}$,where $i \in\{1, \ldots, m\}$. PO informs SUs of this mapping at the beginning of the auction period.

As illustrated above, the PO divides the acquired spectrum opportunity to $S=\left\{s_{1}, s_{2}, \ldots . s_{m}\right\}$ and sells them to $n$ SUs denoted as $U=\{1,2, \ldots, n\}$. The bid submitted by SU $i$ can 


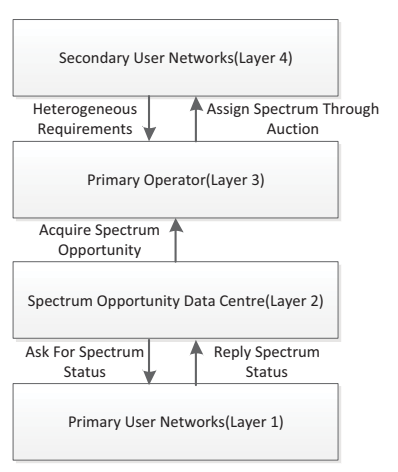

Fig. 2. Cognitive Radio Network Model

be denoted as $b_{i}=\left\{C_{i}, v_{i}\right\}$, where $C_{i} \subseteq S$ and $v_{i}$ is the SU $i$ 's valuation of $C_{i}$. The auction mechanism needs to decide who win the auction and charge SUs to give them incentive to reveal their true valuation of the goods. The winning SUs are denoted as $W=\left\{w_{1}, w_{2}, \ldots, w_{k}\right\}$, where $w_{i} \in U$. The payments of SUs are denoted as $P=\left\{p_{1}, p_{2}, \ldots, p_{n}\right\}$ and auctioneer charges the losing SUs nothing. Therefore, the net utility of $\mathrm{SU} i$ is defined as

$$
u_{i}=\left\{\begin{array}{lll}
v_{i}-p_{i} & i & \text { wins } \\
0 & i & \text { loses }
\end{array}\right.
$$

In the rest of the paper, we use "seller" and "buyer" as the equivalent expressions for PO and SUs, respectively. We will firstly introduce the combinatorial auction model and illustrate how our problem is different from the economical problem.

Definition 1. Combinatorial Auction: In a combinatorial auction, a buyer submits multiple bids to the auctioneer and every bid consists of a bundle of goods he wants to buy and his valuation of that. The final resource allocation must fit two constraints: 1.No good is assigned to more than one buyer; 2. No buyer receives more than one bundle of goods. The combinatorial auction mechanism design consists of two interrelated parts: winner determination and payment mechanism. The task of winner determination is to find the winners, sum of whose declared value(not necessarily be the truthful value) is maximized. The payment mechanism design's goal is to provide a payment policy to charge the winners so that submitting the truthful value to auctioneer for the winner determination is dominating strategy for every buyer. In other words, the payment mechanism guarantees that the winner determination mechanism does maximize the social welfare. The winner determination and payment mechanism design together are called combinatorial auction problem (CAP).

Remarks: We can see from the definition that in general case of combinatorial auction, one buyer only receives at most one bundle of goods but can submit bids on multiple bundles. Different from the general case, in our model, we assume one buyer can submit bid on only one bundle of goods for explicit analysis of the problem. In our future work, we will extend it too the general case.

Definition 2. Cognitive Radio Winning SUs Determination Problem(CRWDP): For every subset $T \subseteq S$, let $b^{j}(T)$ denote the value of buyer $j \in U$ for $T$. We use $e(T, j)=1$ to denote that the buyer $j$ wins the bundle $T$ and $e(T, j)=0$ to denote the buyer loses. The winner determination problem is defined as follows:

$$
\begin{gathered}
\max \sum_{j \in U} \sum_{T \subseteq S} b^{j}(T) e(T, j) \\
\text { s.t. } \sum_{T \ni s_{i}} \sum_{j \in U} e(T, j) \leq 1, \forall s_{i} \in S
\end{gathered}
$$

$$
\sum_{T \subseteq S} e(T, j) \leq 1, \forall j \in U
$$

$\forall i \in U, \exists T_{i}^{*} \subseteq S$ s.t. $b^{i}\left(T_{i}^{*}\right)>0$ and $\forall T \neq T_{i}^{*}, b^{i}(T)=0$.

Remarks:The definition of CRWDP shows that our goal is to maximize the social welfare, that is the sum of winners' value. The first constraint indicates that one good is assigned to only one buyer. The second means one buyer can only have one bundle of goods. The third constraint shows that in this CR network, the buyer can submit up to one bundle of goods, which means he can only choose one bundle of time-frequency slots during this period. Notice that the second and the third constraint seem to be equivalent. However, the second constraint involves with the algorithm design and the third constraint limits the pattern of SUs' bids.

Definition 3. The Truthful Mechanism Design Problem(TMDP): For any buyer $i \in U$, let $b_{i}^{*}=\left\{C_{i}^{*}, v_{i}^{*}\right\}$ denote the truthful bid of the buyer and $b_{i}=\left\{C_{i}, v_{i}\right\}$ denote the declared bid of buyer $i$. Generally, we can assume $C_{i} \supseteq C_{i}^{*}$, because the buyer wants his requirements to be fulfilled. Later in Section III, we can see it is quite straight-forward that $C_{i}=C_{i}^{*}$. As for $v_{i}$, there is no constraint. Further, we use $u_{i}\left(b_{i}^{*}\right)=v_{i}^{*}-p_{i}\left(b_{i}^{*}\right)$ and $u_{i}\left(b_{i}\right)=v_{i}^{*}-p_{i}\left(b_{i}\right)$ to denote the utility when buyer $i$ bids truthfully and bids untruthfully, respectively. The TMDP problem is to design a payment mechanism such that

$$
u_{i}\left(b_{i}^{*}\right) \geq u_{i}\left(b_{i}\right), \forall v_{i} \in R^{+}, C_{i} \supseteq C_{i}^{*}
$$

\section{A Truthful Approximation Auction Mechanism To Solve CAP With TIME-Frequency Flexibility}

\section{A. The Computational Complexity of Optimally Solve CRWDP}

Before we provide our auction mechanism of this problem, we will show that solving CRWDP optimally is NP hard and the upper bound of approximation ratio under polynomial time algorithm is $\sqrt{m}$.

Theorem 1. The CRWDP is NP hard to solve unless $N P=$ $Z P P$.

Proof: We will prove this theorem using reduction from the maximum independent set problem (MISP) [23]. We will build the CRWDP from the MISP. In MISP, we assume the edges are the goods. Therefore, goods set $S$ will now become the edge set $E$ and we use every vertex to denote a buyer. 


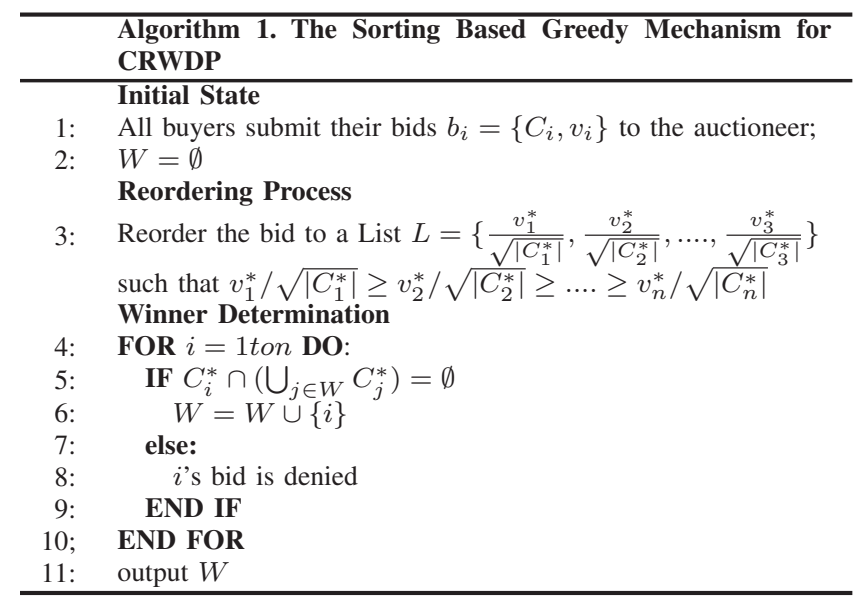

For vertex(buyer) $i$, we assume his interested bundle is the set of his adjacent edges and $v_{i}=1$. For any two winners $w_{i}, w_{j}$ in $W$, their allocated bundles $T_{w_{i}}, T_{w_{j}}$ must satisfy $T_{w_{i}} \cap T_{w_{j}}=\emptyset$. And this indicates that the set of vertex in $W$ is an independent set of the graph. The social welfare is the same as the size of this set. The optimal social welfare is at least $K$ if and only if there exists an independent set of size $K$. Therefore, we reduce MISP,which is NP hard, to CRWDP. From the reduction we can see, the CRWDP is NP hard.

Theorem 2. The upper bound of the approximation ratio of CRWDP is $\sqrt{m}$, for any polynomial time algorithm.

Proof: As shown in theorem 1, the MISP problem can be reduced to CRWDP. Therefore, the approximation ratio of any polynomial algorithm for CRWDP should not exceed the approximation ratio of MISP problem. The upper bound of approximation ratio of MISP is $n^{1-\varepsilon}$ [24]. And in the reduction there is a implicated assumption: $m \leq n^{2}$, and this is easy to be achieved by a proper division of the spectrum opportunity. Therefore, we can conclude that the upper bound of the approximation ratio of CRWDP is $\sqrt{m}$

\section{B. Approximation Method to Solve the CRWDP}

Now we will design an approximation method with the best approximation ratio achievable as described in Theorem 2 . The algorithm is depicted as Algorithm 1.

The algorithm consists of two steps:

- Step1: In the first step, we calculate a norm for every bid $: v_{i} / \sqrt{\left|C_{i}\right|}$ and then reorder the bid by the norm. Without loss of generality, we assume we can have a method to deal with tied bids, for example, randomly arranging, and the final order is descending on the norm $v_{i} / \sqrt{\left|C_{i}\right|}$.

- Step2: A greedy algorithm generates the winner set $W$. The algorithm examines every bid in $L$ sequentially and grants it if it does not overlap with all the previously granted bids. If it does, it will be denied.

We can see that the step 2 is a greedy algorithm and the key part of this algorithm is the first step. Intuitively, we should choose a norm to push the bids, which have a better chance to appear in optimal allocation, towards the beginning of the list $L$. The norm should have two characteristics: 1.we want the bids with higher valuation to have a larger norm; 2.we want the bids with smaller bundle size to have higher norm. From the illustration above we can see that sorting the bids by descending average valuation per good, i.e. $v_{i} /\left|C_{i}\right|$, is a possible choice, but we can generalize the norm to $v_{i} /\left|C_{i}\right|^{l}$ where $l \geq 0$. When we choose $l=1$, the approximation ratio of this algorithm is $m$, which deviates from the upper bound of the possible approximation ratio. As illustrated in algorithm 1 , we choose $l=\frac{1}{2}$ and will prove that the approximation ratio of our algorithm is at least $\sqrt{m}$.

Theorem 3. The proposed algorithm has polynomial-time computational complexity.

Proof: In step 1, the computational complexity of reordering process is $O(n \log (n))$. In step 2, we only need to go over $n$ bids to decide the final allocation. Therefore, computational complexity is $O(n \log (n))$.

Theorem 4. The proposed algorithm can approximate the optimal allocation within a factor of $\sqrt{m}$.

Proof: Recall for every buyer $i \in U$, his bid is denoted as $b_{i}=\left\{C_{i}, v_{i}\right\}$. We now define the cardinality of $C_{i}$ as $c_{i}=\left|C_{i}\right|$ and define the norm as $n_{i}=v_{i} / \sqrt{c_{i}}$. We assume that the $O P T$ is the set of winning bidders in the optimal solution of CRWDP. Then the maximal welfare can be denoted as $v_{o p t}=\sum_{i \in O P T} v_{i}$. Then we denote the solution obtained by our algorithm as $A P P$ and the approximated social welfare is $v_{a p p}=\sum_{i \in A P P} v_{i}$. We now prove that:

$$
v_{o p t} \leq \sqrt{m} v_{a p p}
$$

Without loss of generality, we assume $O P T$ and $A P P$ have no bid in common. If they have, one can remove the common bids. The approximation and optimal solutions of the removed problem are equivalent to the original problem.

Let us consider the expression of $v_{a p p}$ first. We can see that

$$
v_{\text {app }}=\sum_{i \in A P P} v_{i} \geq \sqrt{\sum_{i \in A P P} v_{i}^{2}}=\sqrt{\sum_{i \in A P P} n_{i}^{2} c_{i}}
$$

As for $v_{o p t}$, we have Cauchy-Schwarz inequality such that

$$
v_{\text {opt }}=\sum_{i \in O P T} n_{i} \sqrt{c_{i}} \leq \sqrt{\sum_{i \in O P T} n_{i}^{2}} \sqrt{\sum_{i \in O P T} c_{i}}
$$

The expression $\sum_{i \in O P T} c_{i}$ means the total amount of goods assigned in the optimal allocation and therefore, it is bounded by $m$, the total number of goods.

We then have the following expression:

$$
v_{o p t} \leq \sqrt{\sum_{i \in O P T} n_{i}^{2}} \sqrt{m}
$$

If we want to prove the proposed theorem, it will be enough to prove that:

$$
\sum_{i \in O P T} n_{i}^{2} \leq \sum_{i \in A P P} n_{i}^{2} c_{i}
$$

We then consider the optimal solution $O P T$. As assumed above, there is no bid that appears in both $O P T$ and $A P P$. 
However, the proposed algorithm does go through the whole bids set. So we can conclude that for any $i, i \in O P T$ the following statement stands: when it is considered in the proposed algorithm, there is a good $s_{l} \in C_{i}$ that has already been allocated previously in the approximation algorithm, that is to say, there is a $b_{j} j \in A P P$, where $n_{j}>n_{i}$ and $s_{l} \in C_{j}$.

For any one bid in $A P P$, there can be a number of bids in $O P T$ that fits the above mentioned condition. If for a $b_{i}$, $i \in O P T$, there exists a $j \in A P P$, where $n_{j}>n_{i}$ and $C_{i} \cap C_{j} \neq \emptyset$, we put it in an subset of $O P T$ denoted as $O P T_{j}$. In another word, $O P T_{j}$ denotes all the bids that associate with $j \in A P P$ and a number of different bids from $O P T$ may associate with the same bid $b_{j}$ in $A P P$. However, we should notice that at most $c_{j}$ bids will be associated with $b_{j}$, because no two bids in $O P T$ intersect with each other. Finally we have:

$$
\sum_{i \in O P T_{j}} n_{i}^{2} \leq n_{j}^{2} c_{j}
$$

where $j \in A P P$. Then we have:

$$
\sum_{j \in A P P} \sum_{i \in O P T_{j}} n_{i}^{2} \leq \sum_{i \in A P P} n_{j}^{2} c_{j}
$$

We also have:

$$
\sum_{i \in O P T} n_{i}^{2} \leq \sum_{j \in A P P} \sum_{i \in O P T_{j}} n_{i}^{2}
$$

We combine (10), (11) and (12) so that (9) is proved. The proof is concluded.

Therefore, Our approximation algorithm reaches the best approximation ratio possible for this problem.Moreover, we should notice that the approximation ratio only indicates worst-case scenario. In the simulation part, we will show that the performance difference between optimal solution and approximation solution is very small.

\section{Payment Scheme and Economical Robustness of Proposed Mechanism}

It is critical to notice that the social-welfare calculated in Section III.A is based on the declared bids of buyers. In this part, we will address the other side of the auction mechanism design: TMDP problem, that is to say, we need a payment mechanism to guarantee SUs to submit their true value at the first place.

Definition 4. Payment Scheme: Recall $L$ is the list in which all buyers are reordered by the norm in the first step of algorithm 1. And for buyer $i \in L$, we denote a buyer $l(i)$ as the first buyer following $i$ in $L$ that has been denied but would have been granted were it not for the presence of $i$. We have the following payment:

- $i$ pays zero if his bid is denied or $l(i)$ does not exist.

- If there exists an $l(i)$ and $i$ 's bid is granted, he pays $\sqrt{c_{i}} * n_{l(i)}$, where $n_{l(i)}$ is the norm of $l(i)$.

Before we prove the truthfulness of our payment scheme, we will firstly give a lemma to show the sufficient conditions for a truthful payment mechanism.
Lemma 1. The combinatorial auction for SUs is truthful if and only if the following three conditions hold:

- Ex-post Budget Balance: That means the buyers are all rational so that they will not pay more than their value of the goods.

- Monotonicity: A buyer who wins with $b=\left\{C^{*}, v^{*}\right\}$ can still win with any $v^{\prime} \geq v^{*}$ and any $C^{\prime} \subseteq C^{*}$, when others' bids are fixed.

- Critical Payment: There exists a critical value $v_{c} \leq v_{i}$ for a winner $i$, so that he only needs to pay this critical value to win. That is to say, if others' bids are fixed, the payment of a certain winner does not depend on how he reports his bid.

Proof: We will split the proof to different conditions and, in the following we use $\{C, v\}$ to denote the truthful bid and $\left\{C^{\prime}, v^{\prime}\right\}$ to denote the any other possible bid.

- A truthful buyer will never receive negative utility because his utility is zero while losing and he only needs to pay the critical payment when he wins.

- A winning bidder will never fake bid on the set of goods that he is purchasing. That is to say, he will not be worse off by reporting $\left\{C, v^{\prime}\right\}$ rather than $\left\{C^{\prime}, v^{\prime}\right\}$, where $|C|<\left|C^{\prime}\right|$. Note that $|C|>\left|C^{\prime}\right|$ is not possible because the buyer's requirement will not be fulfilled. Denote the payment for the bid $\left\{C^{\prime}, v^{\prime}\right\}$ by $p^{\prime}$ and for $\left\{C, v^{\prime}\right\}$ by $\mathrm{p}$. For every $q<p$, bidding $\{C, q\}$ will lose since $p$ is a critical value. By monotonicity, $\left\{C^{\prime}, q\right\}$ will also lose for every $q<p$ and therefore, the critical value $p^{\prime} \geq p$. It follows that by bidding $\left\{C, v^{\prime}\right\}$ instead of $\left\{C^{\prime}, v^{\prime}\right\}$, the buyer will still win and his payment will not increase and the net utility remains the same. Therefore, there is no incentive for him to lie.

- We now need to show that no winning buyer will fake bid on the valuation of his bundle of goods. Assume that $\{C, v\}$ is a winning bid with a payment $p_{c}$ (critical value). As long as $v^{\prime}$ is greater than $p_{c}$, the buyer will still win with the same payment. When $v^{\prime}<p_{c}$, the buyer gains zero utility because he will lose the auction. Thus, misreporting his value will not be beneficial.

- The previous two paragraphs show the cases of winners faking the bid. As for the losers, it is obvious that if they report their value higher to win, the payment will exceed their valuation and thus they receive negative utility. And it is not possible for them to reduce the size of the bundle they are bidding for because it is meaningless to obtain goods less then their requests.

Theorem 5. The proposed auction mechanism is a truthful mechanism.

Proof: According to Lemma 1, we only need to verify that our mechanism satisfies the three properties. Ex-post budget balance is guaranteed in our auction mechanism because we assume the SUs are rational buyers. Monotonicity also stands because by increasing value or decreasing the number of desired goods, the SU's position in $L$ can only move forward 
and make it easier to win. The critical payment condition is met because $i$ wins as long as he appears in $L$ before $l(i)$. The payment is exactly at the transition point between $i$ being before and after $l(i)$. Therefore, we can conclude from the Lemma 1 that our auction mechanism is a truthful one, that is to say no participant in our auction will lie about his true valuation and the economical robustness is guaranteed.

\section{Optimal and Truthful Auction Mechanism for a Restricted Case of the General Model}

\section{A. A Rational Modification of the System Model}

As illustrated in section III, under the time-frequency two dimensional flexibility, no optimal solution can be found in polynomial time and only approximation algorithm with approximation ratio no better than $\sqrt{m}$ can be found. In this section, we will modify the general system model presented in section II to a little more restricted, but still useful, model and design an optimal and truthful auction mechanism under the modified model.

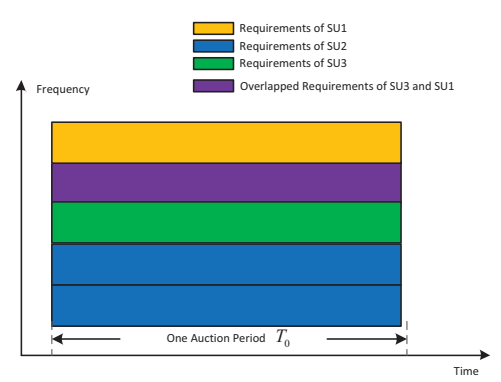

Fig. 3. Modified System Model with Only Frequency Flexibility

We will firstly illustrate how the model is changed. As shown in Figure 3, two assumptions are different from the general model.

- Full Time Usage: In the first place, the SUs cannot choose to use partial time of the whole auction period but have to buy full-time usage or nothing.

- Consecutive Requirement: In the second place, the SU can only use adjacent frequencies, that is to say, for any $\mathrm{SU}$, the desired frequency is an interval $\left[f_{0}, f_{1}\right]$, but not $\left[f_{0}, f_{1}\right]$ and $\left[f_{2}, f_{3}\right]$, where $\left[f_{0}, f_{1}\right] \cap\left[f_{2}, f_{3}\right]=\emptyset$.

We do realize that the first restriction undermines the flexibility of our mechanism. However, it is a necessary tradeoff to reach the optimality. The rationale behind the second restriction is that we assume SUs are all single-antenna devices, which is common in today's mobile devices. The reason why we do not use the single-antenna assumption in the general model is that it will make the CRDWP more complicated to solve. However, under the restricted model, this consecutive requirements nature makes the optimal solution easy to reach. What's more, the application meaning of the model is still acceptable. In our model, PO can divide the frequency he obtained without so much concern about the requirements of
SUs. For example, in the previous auction models [18] [16], the auctioneer must decide the bandwidth to conduct a auction. If auctioneer chooses the auctioned bandwidth to be too large, this choice may exceed the requirements of most SUs and no one is willing to pay high for the too large spectrum and thus reduce the profits of PO. However, in our model, the auctioneer can just divide all spectrum into reasonably thin slices and list them to SUs like a supermarket. SUs can freely choose any consecutive combination of frequency to form whatever large or small bandwidth they need. We now define the modified model as follows:

In the modified DSA model, the PO owns the spectrum opportunity and divides it only by frequency, the goods set is redefined as $S=\left\{f_{1}, f_{2}, \ldots, f_{m}\right\}$, where $f_{i}$ is the starting frequency point of the $i$ th interval: $\left[f_{i}, f_{i}+\Delta f\right]$, and $f_{1} \leq f_{2} \leq \ldots . \leq f_{m}$. Because in this model, only consecutive frequency can be required, we redefine the $C_{i}$ as a close interval $C_{i}=[r, q]$, which means the $\mathrm{SU}$ wants the frequency that starts from $f_{r}$ and ends at $f_{q}$. Further, we give a new notation: $h\left(\left[f_{i}, f_{j}\right]\right)$ to denote the highest valuation submitted to auctioneer on $\left[f_{i}, f_{j}\right]$. If there is no bid on $\left[f_{i}, f_{j}\right]$ we will set it to 0 . Moreover, we define $G=\{T \mid T \subseteq S$ and $T$ fits the Consecutive Requirement condition $\}$. Then we give a more succinct definition of the CRWDP, as CRWDP-C (Constrained).

Definition 5 CRWDP-C: The problem of CRWDP-C can be formulated as:

$$
\begin{gathered}
\max \sum_{T \subseteq G} h(T) e_{T} \\
\text { s.t. } \sum_{T \ni f_{i}} e_{T} \leq 1, \forall f_{i} \in S \\
e_{T}=\{0,1\}, \forall T \in G
\end{gathered}
$$

$\forall i \in U$ there is one and only $T_{i}^{*} \subseteq S$ s.t. $b^{i}\left(T_{i}^{*}\right)>0$, for all other $T \neq T_{i}^{*}, b^{i}(T)=0 . e_{T}=1$ means that the highest bid on $T$ will be accepted in the final allocation and $e_{T}=0$ means it will not.

The TMDP is not changed and we will tackle them separately in the following.

\section{B. Optimal Solution for CRWDP-C with a Dynamic Program- ming Algorithm}

Different from CRWDP, the CRWDP-C can be solved optimally in polynomial time. We will give our algorithm first and prove the optimality and computational efficiency. We use $h([i, j])$ to denote $h\left(\left[f_{i}, f_{j}\right]\right)$ for simplicity and define a function whois $(h[i, j])$ to return the buyer who submits the highest value on $[i, j] . O P T_{a}$ denotes all the intervals that are allocated in the optimal allocation and $W$ denotes all the winners. Further, we use $v_{\text {opt }}$ to denote the optimal social welfare. Algorithm 2 generates the optimal solution of CRWDP-C and the basic idea behind the algorithm is that the maximal social welfare of first $k+1$ frequency slices can be evaluated by evaluating the maximal social welfare of first $l$ 


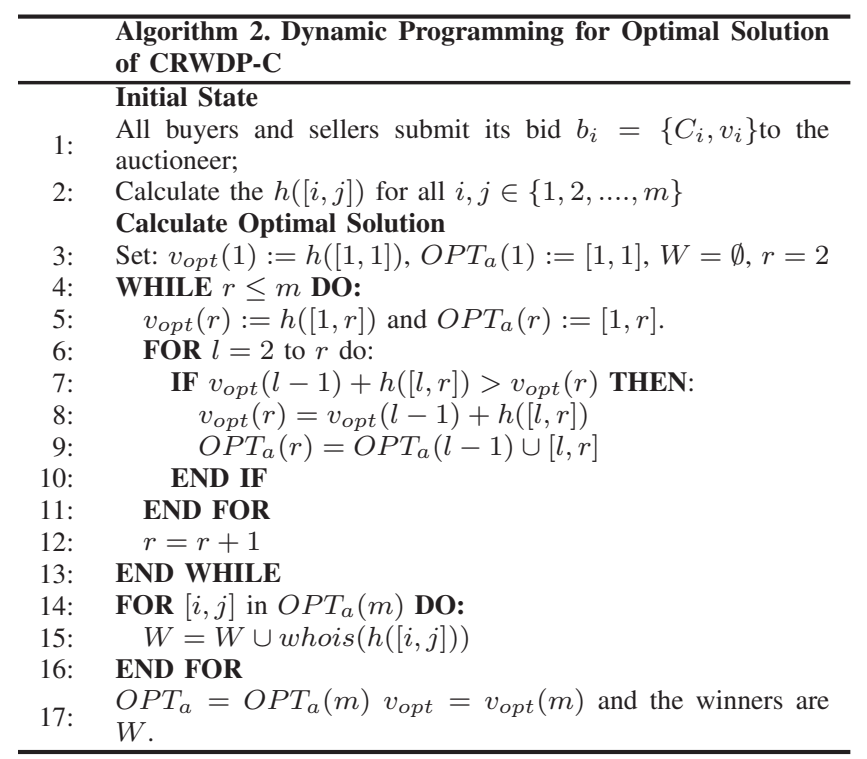

frequency slices and adding $h([l+1, k+1])$. If we have $v_{\text {opt }}$ for all $l \leq k$ and go over all $0 \leq l \leq k$, we can calculate $v_{\text {opt }}$ for $k+1$ frequency slices.

Theorem 6 Algorithm 2 achieves optimal solution $O P T_{a}$ for CRWDP-C and the computational complexity is $O\left(n^{2}\right)$.

Proof: The computational complexity for this algorithm is obviously $O\left(n^{2}\right)$. For the optimality, we will do induction on the number of frequency slices $m$ to prove $O P T_{a}$ is an optimal allocation.

- When $m=1$, it is clear that $O P T_{a}(m)=\{[1,1]\}$ is an optimal solution.

- Suppose that $\forall m \leq k O P T_{a}(m)$ is an optimal solution for the auction of the first $m$ frequency slices. We will consider if the algorithm 2 generates optimal allocation for $m=k+1$ frequency slices. Because of the consecutive requirement property of this model, if the $k+1$ th frequency slice is included in the optimal solution, it must be in the form of $[j+1, k+1]$ where $j \leq k$. As assumed, we have $O P T_{a}(j)$ an optimal allocation for first $j$ frequency set. Then we have $O P T_{a}(k+1)=O P T_{a}(j) \cup[j+1, k+1]$ and $v_{\text {opt }}(k+1)=v_{\text {opt }}(j)+h([j+1, k+1])$. Algorithm 2 searches every possible $j$ and will find the optimal allocation for the first $k+1$ frequency slices under the induction assumption.

- Based on the induction hypothesis, we can conclude that $O P T_{a}(m)$ is the optimal solution for the whole $m$ frequency slices auction.

Therefore, we solve the CRWDP-C optimally in polynomial time and we will begin to design the payment scheme to guarantee the economical truthfulness of the auction in modified model.

C. Truthful Payment Scheme for the Combinatorial Auction in Modified System Model

As illustrated in section I, only a truthful auction is a meaningful auction. For the combinatorial auction in the mod- ified system model, we will use generalized VCG mechanism proposed by Clarke[25] and Groves[26] to design a truthful payment mechanism. Clarke and Groves showed that if in an auction mechanism the winner determination can be solved optimally, that is to say, the final allocation can maximize the social welfare, then for a certain bidder, the payment should be the sum of the declared valuation of other bidders minus the sum of such valuation that would have been obtained if he had not participated. In another word, the payment is externality of the buyer on the auction. Under this payment scheme, the truthfulness of auction can be guaranteed. We will apply the generalized VCG mechanism to our TMDP in the restricted model and give definition of a buyer $i$ 's payment as follows:

Definition 6.VCG Payment Mechanism for Proposed Combinatorial Auction: We denote the payment for buyer $i$ as $p_{i}$. We denote $g_{i}$ as the goods buyer $i$ receives in the final allocation. We can see that $g_{i}=C_{i}$ when buyer $i$ wins and $g_{i}=\emptyset$ when $i$ loses. Further, we denote $v\left(g_{i}\right)$ as valuation obtained by buyer $i$ in the final allocation. Note that $v\left(g_{i}\right)=v_{i}$ when $i$ wins and $v\left(g_{i}\right)=0$ when $i$ loses. We denote $v_{o p t-i}$ as the optimal social welfare obtained in the auction where $i$ is absent. Note that despite $i$, all other buyers stay the same when calculating $v_{o p t}$ and $v_{o p t-i}$. We have that the payment is:

$$
p_{i}=v_{\text {opt-i }}+v\left(g_{i}\right)-v_{\text {opt }}
$$

We now give the proof of truthfulness of our payment scheme:

Theorem 7 The VCG payment mechanism guarantees truthfulness of the overall auction mechanism.

Proof: The proof the truthfulness of the generalized VCG mechanism is illustrated very clearly in [27]. Therefore, we refer readers to this book and will not repeat the proof in our paper.

Remarks: A natural question raises now: since there is a standard truthful payment design mechanism, why do we not use it in section III? The reason is that generalized VCG mechanism had certain limitations, because the payment of VCG mechanism is calculated based on the optimal social welfare and we have proved that the optimal social welfare cannot be calculated in polynomial time under the model of section III.

\section{Simulation Results}

\section{A. Simulation of the Time-Frequency Two Dimensional Flex- ibility Model}

We assume there is only one primary operator in the network and the auction period is one hour. The primary operator divides the auction period into 10-min long 6 sectors and divides the whole frequency band to 24 slices. Therefore, the number of frequency-time slots for SUs to choose is $m=144$. We assume the number of SUs $n$ varies from 100 to 1000 . The maximal requirement for any SU is 20 slots. We further assume that the SUs' values are uniformly distributed in $[0,1]$. 


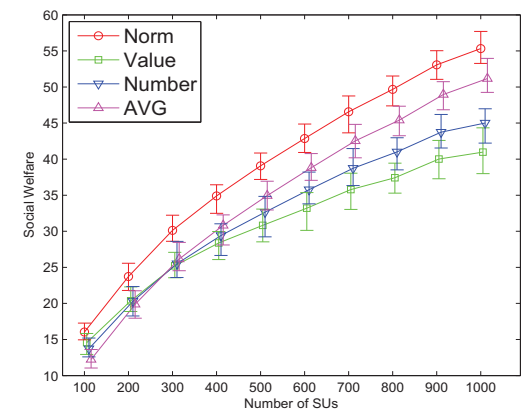

Fig. 4. Comparison of social welfare under general model.

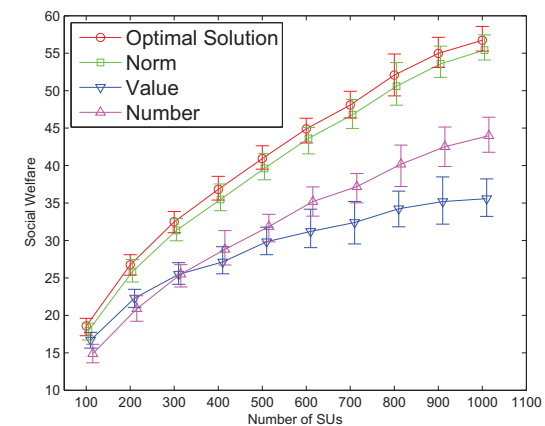

Fig. 7. Comparison of social welfare under restricted model.

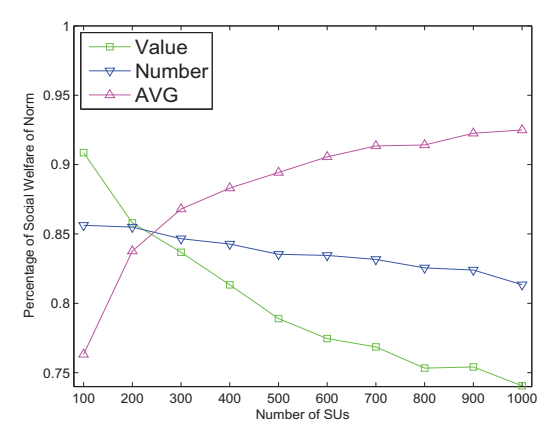

Fig. 5. Comparison of social welfare under general model using norm as a benchmark.

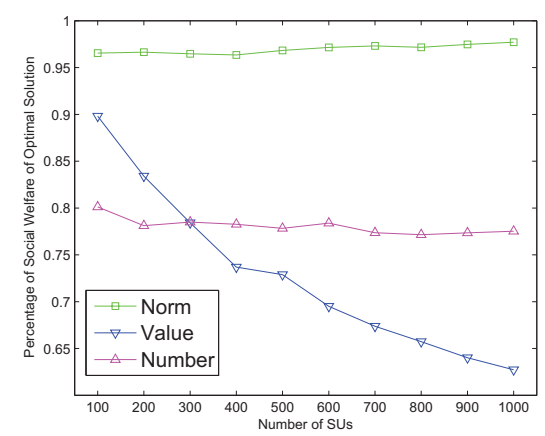

Fig. 8. Comparison of social welfare under general model using optimal solution as a benchmark.

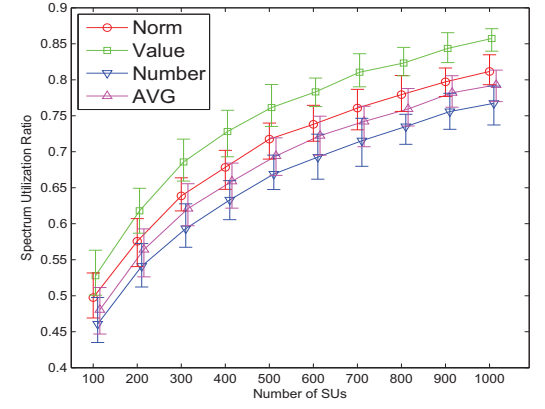

Fig. 6. Comparison of utilization ratio under general model.

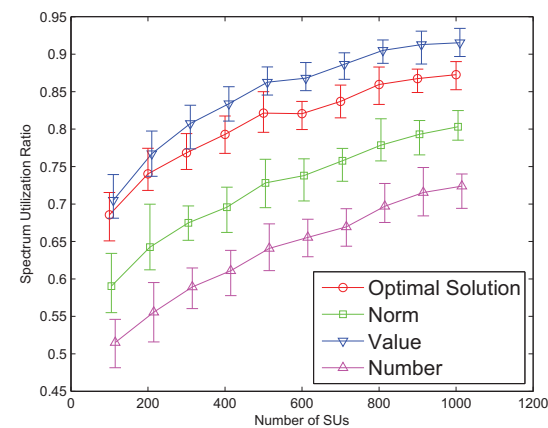

Fig. 9. Comparison of spectrum utilization ratio under restricted model.
In Figure 4, we depict the relation between social welfare and the number of SUs. We use different ordering methods and the greedy algorithm to generate allocation results. And in this simulation, we assume that every slot required by SUs is randomly distributed among 1 to $m$ in a uniform manner. As we can see, the social welfare generated under the proposed ordering method, denoted as Norm, is superior to all other intuitive methods. Value means reordering the bids only in descending order of valuation. Number means reordering the bids only in ascending order of the number of the required slots. $A V G$ means reordering the bids in descending order of average value per slot. In Figure 5, we use Norm as a benchmark to evaluate percentage of social welfare reached by other algorithms compared to that reached by Norm. We can see that as the SUs' number increases, the performance of Number and Value are degrading and that of $A V G$ becomes better.

In this part, we cannot give a numerical comparison between the optimal and the approximation solution because solving CRWDP optimally is NP hard. However, there is only 144 slots and the maximal value for any bundle is 1 . Therefore, the optimal solution is no larger than 144. As we have illustrated in Section III, our algorithm's worst case approximation ratio is $\sqrt{m}$, that is $\frac{1}{12}$. However, we can see that the social welfare obtained by our algorithm is much larger than $\frac{1}{12} \times 144=12$. Our algorithm performs much better than worst case ap- proximation ratio. A more explicit and numerical comparison between optimal and approximation method will be done in the simulation of the restricted network model.

In Figure 6, we show the spectrum utilization ratio, which indicates the ratio of allocated slots to the total slots of PO. Figure 6 shows that Value has the best spectrum utilization ratio and Norm is the second best followed by $A V G$ and Num. We can see that to reach a better social welfare, the utilization ratio is compromised. However, when the number of SUs is sufficiently large, we can see that the utilization ratio of Norm is acceptable.

\section{B. Simulation of the Restricted Model}

In this subsection, we move to the restricted model in Section IV. We assume that the primary operator divides the spectrum opportunity into $m=144$ frequency slices. The SUs' requirements must meet consecutive requirements and full time usage condition. Therefore, we can generate an SU's requirement by a start point of frequency and a interval length. In this simulation, a SU can require at most 20 consecutive frequency slices. The start points of his requirement are uniformly distributed in 1 to 144 .

It is critical to notice that the CRWDP-C is a special case of CRWDP. Though we have polynomial time optimal algorithm for CRWDP-C, the approximation method for CRWDP can also be implemented in CRWDP-C. Therefore, it is actually 
a good chance to test the performance of the approximation algorithm. In Figure 7, we not only depict the optimal social welfare against the number of SUs but also give the results of other three approximation methods. In Figure 8, we use the optimal solution as a benchmark to evaluate other three approximation algorithms. The algorithm Norm, which is proposed in Section III, continually has a high approximation ratio, around $97 \%$ of the optimal solution. Therefore, we can conclude that our approximation algorithm performs very well.

In Figure 9, we show the utilization ratio of different algorithms in the modified model. The utilization ratio of the optimal solution is not the highest. We regard this as a necessary tradeoff to achieve high social welfare. We also observe that the utilization ratio of the proposed approximation algorithm is not as high as that of the optimal algorithm. However, the utilization ratio is sufficiently high when the number of SU is large.

\section{CONCLUSiOn AND Future WORKS}

In this paper, we firstly design a novel model to divide the spectrum opportunity of primary operator into time-frequency slots. Under this model, to cater to the time-frequency flexible requirements, we design a truthful and efficient combinatorial auction method to approximate the optimal solution of social welfare, which is NP hard to solve. Then we reduce the time-frequency two dimensional flexibility to only frequency flexibility. Under the restricted model, we devise a polynomial time winner determination algorithm to reach the optimal social welfare and a VCG payment mechanism to guarantee the truthfulness. In simulation, we show that our algorithms perform well and shows a tradeoff between social welfare and spectrum utilization ratio.

Because we model the SUs' requirements in a new way and introduce the concept of combinatorial auction into cognitive radio. We believe there are many future works to be done. Firstly, in our paper, we only allow one SU submits one bid $b=\{C, v\}$ at a time. If we allow one $\mathrm{SU}$ to submit multiple bids at a time, that is to say, if any of these bids is fulfilled, the requirement of this $\mathrm{SU}$ can be regard as fulfilled. The whole system will have more application meanings. What's more, an online auction mechanism with consideration of timefrequency flexibility is also an interesting topic.

\section{ACKNOWLEDGMENT}

This work is supported by National Fundamental research grant (2011CB302701), NSF China (No. 60832005); China Ministry of Education New Century Excellent Talent (No. NCET-10-0580); China Ministry of Education Fok Ying Tung Fund (No. 122002); Qualcomm Research Grant; Shanghai Basic Research Key Project (No.11JC1405100); National Key Project of China (2010ZX03003-001-01).

\section{REFERENCES}

[1] T. Rappaport, Wireless communications. Prentice Hall, 2002.

[2] M. Buddhikot and K. Ryan, "Spectrum management in coordinated dynamic spectrum access based cellular networks," in Proc. IEEE DySPAN,, Baltmore, USA, Nov. 2005.
[3] F. Force, "Report of the spectrum efficiency working group," Washington DC, noviembre de, 2002.

[4] J. Mitola, "Cognitive radio: making software radios more personal," IEEE Journal on Personal Communications, vol. 6 no. 4 pp.13-18, Aug. 1999.

[5] L. Duan, J. Huang and B. Shou, "Investment and Pricing with Spectrum Uncertainty: A Cognitive Operator's Perspective," IEEE Transactions on Mobile Computing, vol. 10, no. 11, pp. 1590-1604 Nov. 2011.

[6] W. Saad, "Coalitional Game Theory for Distributed Cooperation in Next Generation Wireless Networks," Ph.D. dissertation, University of Oslo, 2010.

[7] L. Gao, X. Wang, Y. Xu, and Q. Zhang, "Spectrum trading in cognitive radio networks: A contract-theoretic modeling approach," IEEE Journal on Selected Areas in Communications, vol. 29, no. 4, pp. 843-855, 2011.

[8] J. Huang, R. Berry, and M. Honig, "Auction-based spectrum sharing," ACM Journal on Mobile Networks and Applications, vol. 11, no. 3, pp. 405-418, 2006.

[9] V. Krishna, Auction theory. Academic press, 2009.

[10] G. Kasbekar and S. Sarkar, "Spectrum auction framework for access allocation in cognitive radio networks," IEEE/ACM Transactions on Networking , vol. 18, no. 6, pp. 1841-1854, 2010.

[11] S. Sodagari, A. Attar, and S. Bile?n, "On a truthful mechanism for expiring spectrum sharing in cognitive radio networks," IEEE Journal on Selected Areas in Communications, vol. 29, no. 4, pp. 856-865, 2011.

[12] P. Xu and X. Li, "Tofu: Semi-truthful online frequency allocation mechanism for wireless networks," IEEE/ACM Transactions on Networking, vol. 19, no. 2, pp.433-446, Apirl 2011.

[13] J. Huang, Z. Han, M. Chiang, and H. Poor, "Auction-based resource allocation for cooperative communications," IEEE Journal on Selected Areas in Communications, vol. 26, no. 7, pp. 1226-1237, 2008.

[14] A. Gopinathan, Z. Li, and C. Wu, "Strategyproof auctions for balancing social welfare and fairness in secondary spectrum markets," in Proc. IEEE INFOCOM, Shanghai, China, April 2011.

[15] S. Wang, P. Xu, X. Xu, S. Tang, X. Li, and X. Liu, "Toda: Truthful online double auction for spectrum allocation in wireless networks," in Proc. IEEE DySPAN. Singapore, Apirl 2010,

[16] L. Gao, Y. Xu and X. Wang, "MAP: Multi-Auctioneer Progressive Auction for Dynamic Spectrum Access," IEEE Transactions on Mobile Computing , vol.10, no.8, pp.1144-1161, 2011.

[17] S. Gandhi, C. Buragohain, L. Cao, H. Zheng, and S. Suri, "A general framework for wireless spectrum auctions," in Proc. IEEE DySPAN, Dublin, Ireland, Apirl 2007.

[18] L. Chen, S. Iellamo, M. Coupechoux, and P. Godlewski, "An auction framework for spectrum allocation with interference constraint in cognitive radio networks," in Proc. IEEE INFOCOM. San Diego, USA, March 2010.

[19] L. Duan, J. Huang and B. Shou, "Cognitive mobile virtual network operator: Investment and pricing with supply uncertainty," in Proc. IEEE INFOCOM. San Diego, USA, March 2010.

[20] P. Xu, X. Xu, S. Tang, and X.-Y. Li, "Truthful online spectrum allocation and auction in multi-channel wireless networks," in Proc. IEEE INFOCOM, Shanghai, China, April 2011.

[21] L. Deek, X. Zhou, K. Almeroth, and H. Zheng, "To preempt or not: Tackling bid and time-based cheating in online spectrum auctions," in Proc. IEEE INFOCOM, Shanghai, China, April 2011.

[22] P. Cramton, Y. Shoham, and R. Steinberg, "Combinatorial auctions," 2006.

[23] J. Bondy and U. Murty, Graph theory with applications. MacMillan London, 1976, vol. 290.

[24] J. Hastad, "Clique is hard to approximate within n1-ع," in Proc. IEEE FOCS, Burlington, USA, Oct. 1996.

[25] E. Clarke, "Multipart pricing of public goods," Journal on Public choice, vol. 11, no. 1, pp. 17-33, 1971.

[26] T. Groves, "Incentives in teams," Journal of the Econometric Society, pp. 617-631, 1973.

[27] N. Nisan, Algorithmic game theory. Cambridge Univ Pr, 2007.

[28] S. Dobzinski and N. Nisan, "Limitations of vcg-based mechanisms," in Proc. ACM symposium on Theory of computing. San Diego, USA, June 2007.

[29] Y. Liu, K. Liu and M. Li, "Passive diagnosis for wireless sensor networks," IEEE/ACM Transactions on Networking. vol. 18, no. 4, pp. 1132-1144, 2010. 